\title{
Magnetic properties study of iron-oxide nanoparticles/PVA ferrogels with potential biomedical applications
}

\author{
P. Mendoza Zélis $\cdot$ D. Muraca $\cdot$ J. S. Gonzalez \\ G. A. Pasquevich • V. A. Alvarez • K. R. Pirota • \\ F. H. Sánchez
}

Received: 11 January 2013/ Accepted: 26 March 2013

(C) Springer Science+Business Media Dordrecht 2013

\begin{abstract}
A study of the magnetic behavior of maghemite nanoparticles (NPs) in polyvinyl alcohol (PVA) polymer matrices prepared by physical crosslinking is reported. The magnetic nanocomposites (ferrogels) were obtained by the in situ co-precipitation of iron salts in the presence of PVA polymer, and subsequently subjected to freezing-thawing cycles. The magnetic behavior of these ferrogels was compared with that of similar systems synthesized using the glutaraldehyde. This type of chemical cross-
\end{abstract}

Electronic supplementary material The online version of this article (doi:10.1007/s11051-013-1613-6) contains supplementary material, which is available to authorized users.

P. Mendoza Zélis $(\bowtie) \cdot$ F. H. Sánchez

IFLP-CONICET and Departamento de Física,

Universidad Nacional de La Plata, Casilla de Correos 67, 1900 La Plata, Argentina

e-mail: pmendoza@ fisica.unlp.edu.ar

D. Muraca · K. R. Pirota

Gleb Wataghin Physics Institute, Universidade Estadual

de Campinas, Rua Sergio Buarque de Holanda,

777 Cidade Universitaria, Zeferino Vaz Barao Geraldo,

Campinas, São Paulo CEP 13083-859, Brazil

e-mail:dmuraca@ifi.unicamp.br

J. S. Gonzalez · V. A. Alvarez

COMP-INTEMA-CONICET and Universidad de Mar del

Plata, Solís 7575, 7600 Mar del Plata, Argentina

G. A. Pasquevich

IFLP-CONICET and Facultad de Ingeniería, La Plata, Argentina linking agents presents several disadvantages due to the presence of residual toxic molecules in the gel, which are undesirable for biological applications. Characteristic particle size determined by several techniques are in the range $7.9-9.3 \mathrm{~nm}$. The iron oxidation state in the NPs was studied by X-ray absorption spectroscopy. Mössbauer measurements showed that the NP magnetic moments present collective magnetic excitations and superparamagnetic relaxations. The blocking and irreversibility temperatures of the NPs in the ferrogels, and the magnetic anisotropy constant, were obtained from magnetic measurements. An empirical model including two magnetic contributions (large NPs slightly departed from thermodynamic equilibrium below $200 \mathrm{~K}$, and small NPs at thermodynamic equilibrium) was used to fit the experimental magnetization curves. A deviation from the superparamagnetic regime was observed. This deviation was explained on the basis of an interacting superparamagnetic model. From this model, relevant magnetic and structural properties were obtained, such as the magnitude order of the dipolar interaction energy, the NPs magnetic moment, and the number of NPs per ferrogel mass unit. This study contributes to the understanding of the basic physics of a new class of materials that could emerge from the PVA-based magnetic ferrogels.

Keywords Nanoparticles · Polymer-matrix composites - Magnetic properties · Ferrogel · Maghemite 


\section{Introduction}

Nanostructured materials are of great technological importance in the design of new functional and multifunctional devices. The large number of applications for these nanostructured materials makes the understanding of the physics behind their behavior of practical and fundamental relevance. Nanostructured magnetic systems are composed of objects with at least one dimension in the nanometric scale, and display novel properties as a direct result of their small size. One type of these systems, known as colloids, has nanoparticles (NPs) as its main component. Colloids systems are made of NPs dispersed in solids (Sholklapper et al. 2008), liquids (e.g., ferrofluids) (Chandrasekar et al. 2012), and in soft gel matrices (e.g. ferrogels) (Schexnailder and Schmidt 2009), among others.

In part, the relevance of nanostructured materials comes from the possibility of miniaturizing devices and increasing their number of applications. However, their main technological and scientific impacts come from the fact that their nanoscale size results in confinements, single-domain states and high surface to volume ratios. Some magnetic materials are composed of iron-oxide NPs. The most commonly used iron oxides are the maghemite $\left(\gamma-\mathrm{Fe}_{2} \mathrm{O}_{3}\right)$ and the magnetite $\left(\mathrm{Fe}_{3} \mathrm{O}_{4}\right)$. Some magnetic materials are composed of iron-oxide NPs. The most commonly used iron oxides are the maghemite and the magnetite. Because of their high level of biocompatibility, interest in them for their biomedical applications grows continuously. These materials can be used as agents in magnetic resonance imaging contrast enhancement (Masoudi et al. 2012), in clinical trials for drug delivery (Zhao et al. 2012), in hyperthermia tumor treatment under radiofrequency fields (Pankhurst et al. 2009; Rivas et al. 2012), among others (Knobel et al. 2008; Krishnan 2010; Serantes et al. 2010a). Magnetic NPs can be introduced into a soft gel matrix making a new class of magnetocontrolled elastic material, known as ferrogel. The coupling between the soft elastic medium and the magnetic NPs allows the manipulation of the volume and shape of the ferrogels through the application of external magnetic fields. The effects of the magnetic fields on strain (Faidley et al. 2010), shape (Raikher and Stolbov 2005; Zrínyi et al. 1997), water retention (Chen et al. 2005; François et al. 2007), stiffness (Mitsumata et al. 1999), and the effect of the polymeric matrix on the structural and magnetic properties
(Longo et al. 2012) have been reported. Because of these properties, ferrogels have many potential applications in the fields of pharmaceutics and medicine as biomembranes, biosensors, artificial muscles, and matrices for drug delivery, among others (Furukawa et al. 2003; Gonzalez et al. 2012; Liu et al. 2006; Mao et al. 2005; Miyata et al. 2002; Pich et al. 2004; Qiu and Park 2001; Sivudu and Rhee 2009).

In general, these ferrogels are made by incorporating magnetic NPs into polymeric hydrogels. Polyvinyl alcohol (PVA) is a hydrophilic polymer from which biocompatible, elastic and mechanically strong hydrogels can be obtained. This polymer can be prepared by means of different techniques, such as chemical crosslinking (Matsuyama et al. 1997) (chemical agents), radiation cross-linking (Hill et al. 2011) (electron beam, $\gamma$-irradiation), and physical cross-linking (Hassan and Peppas 2000) (freezing-thawing).

The main advantages that freezed-thawed gels present over gels synthesized with other techniques [i.e., chemical cross-linking using a cross-linking agent such as glutaraldehyde (GA) (Matsuyama et al. 1997), photo-polymerization by UV radiation (Mawad et al. 2009), electron irradiation (Bray and Merrill 1973)] are: superior physical and mechanical properties (Hassan and Peppas 2000; Yang et al. 2008), simple preparation technique, and no leachable toxic synthesis waste which can be harmful to the human body (Peppas and Mongia 1997). Chemical cross-linking agents, such as GA, could present certain disadvantages associated with the presence of residual amounts of toxic molecules in the gel, which are undesirable for biomedical applications (Peppas and Mongia 1997). The use of complex irradiation techniques, such as electron beam or $\gamma$-irradiation, usually causes problems associated with bubble formation (Cima and Lopina 1995). In the physical cross-linking process known as freezingthawing (FT), the crystallites formed during FT cycles act as the cross-linking points of the gel matrix. These materials exhibit higher mechanical strength than gels obtained by chemical or irradiation techniques, and are easier to prepare (Hassan and Peppas 2000).

The study of the magnetic properties of ferrogels and the understanding of the phenomena experienced by the ferrogels under magnetic fields are necessary for the future development and application of these materials. In systems where there is NP interaction, the dependence of the magnetic properties on the magnetic field $H$ and the temperature $T$ is an old but still 
open question for debate among the scientific community. Moreover, NP spatial distribution, size distribution, interaction and potential aggregation in PVA polymeric matrices have not yet been completely described. Recently, P. Allia and P. Tiberto have published an interesting work in which they study the limits of the superparamagnetic regime in the presence of dipolar magnetic NP interactions in a polymeric matrix (Allia and Tiberto 2011), based on a previous work devoted to Co-Cu alloys (Allia et al. 2001). As regards the ferrogels described in this work, even less studies about their detailed magnetic properties, especially about how dipolar interactions influence the determination of specific quantities like the particle magnetic moment, are available. Different studies have focused on the drug release behavior (Liu et al. 2006) and on the changes in stress (Mitsumata et al. 1999) under magnetic fields. More recently, Hoppe et al. (2008) have reported studies on magnetic NPs synthesized and dispersed in polymeric matrices, in which they suggest the existence of a thermodynamic spin-glass phase due to dipolar interactions in the materials.

In this work, we study ferrogels obtained by the synthesis of iron-oxide NPs in a PVA-containing solution by means of the co-precipitation technique and the subsequent physical cross-linking of the polymer through FT cycles, and present an in-depth characterization of the magnetic properties of these ferrogels. These properties are compared with those of GA chemically cross-linked ferrogels, which are also reported here. Based on the magnetic properties at different temperatures, a superparamagnetic interaction model was used to better understand the physics behind the magnetic measurements. From a technological point of view, the obtained results are very promising, and represent progress on the physical knowledge of superparamagnetic systems with dipolar interactions.

\section{Materials and methods}

PVA was dissolved in a solution containing $\mathrm{Cl}_{3} \mathrm{Fe} \cdot 6 \mathrm{H}_{2} \mathrm{O}, \mathrm{SO}_{4} \mathrm{Fe} \cdot 7 \mathrm{H}_{2} \mathrm{O}(2: 1)$ and $\mathrm{HCl}$ [used to avoid oxidation of $\mathrm{Fe}$ (II)] under slow magnetic stirring at $85^{\circ} \mathrm{C}$ for $4 \mathrm{~h}$. The ratio of $\mathrm{Fe}^{2+} / \mathrm{Fe}^{3+}$ solutions was selected to obtain NP-PVA mass nominal relation of 6 wt $\%$. After cooling to room temperature, $\mathrm{NH}_{3}$ was added to the clear solution to trigger the co-precipitation of iron-oxide NPs. The final $\mathrm{pH}$ value of this mixed solution was 10 (Jolivet et al. 2004; Kim et al. 2003; Liang et al. 2006). Thin samples of the ferrogels were obtained by cross-linking on at molds using aliquots of the initial solution. The FT samples were obtained by freezing the solution for at $-18{ }^{\circ} \mathrm{C}$ for $1 \mathrm{~h}$, and then heating it at $25{ }^{\circ} \mathrm{C}$ for the same period of time (thawing process). This FT cycle was repeated several times in order to trigger the cross-linkings. The GA samples were obtained by mixing $2.8 \mathrm{~mL}$ of solution of methanol (3 mL, $50 \mathrm{vol} \%)$, acetic acid ( $2 \mathrm{~mL}, 10 \mathrm{vol} \%$ ) and GA (1 mL, 5 vol\%); adding it to the initial $10 \mathrm{~mL}$ of PVA solution; and then casting it into an anti-adherent container. In both cases, the resulting films were dried until constant weight was reached $\left(85^{\circ} \mathrm{C}, 24 \mathrm{~h}\right)$. PVA with an average molecular weight of $93,000 \mathrm{~g} / \mathrm{mol}$ and a hydrolysis degree higher than $98-99 \%$ was purchased from Sigma-Aldrich (Germany). Iron (II) sulfate, heptahydrated, and Iron (III) chloride, hexahydrated, were purchased from Cicarelli laboratory (Argentine), hydrochloric acid was from Biopack (Argentine) and ammonium hydroxide was provided by Anedra laboratory (Argentine). Details of the synthesis and reactions are described in Gonzalez et al. (2011).

The identification of the $\mathrm{Fe}$ oxidation state was performed by X-ray absorption near edge spectroscopy (XANES) measurements. These measurements were made at XAFS2 beamline of LNLS (Campinas, Brazil). XANES and EXAFS spectra of the Fe $K$-edge $(7,112 \mathrm{eV})$ were recorded at room temperature in transmission mode using a $\mathrm{Si}$ (111) monochromator.

The $14.4 \mathrm{keV}\left(I_{\mathrm{g}}=\frac{1}{2}, M 1, I_{\mathrm{e}}=\frac{3}{2}\right)^{57} \mathrm{Fe}$ Mössbauer spectra were acquired using a ${ }^{57} \mathrm{Co}(\mathrm{Rh})$ source at $295 \mathrm{~K}$ in transmission geometry, with a standard spectrometer operating in the constant acceleration regime. The isomer shifts were measured in relation to $\alpha$-Fe at $295 \mathrm{~K}$. The ferrogel absorber thickness was $350 \mathrm{mg} / \mathrm{cm}^{2}$. The number of channels used for transmission data recording versus source-absorber velocity was 1,024 . The spectrometer line width with a $13 \mu \mathrm{m} \alpha$-Fe absorber was $0.21 \mathrm{~mm} / \mathrm{s}$.

The magnetic properties (mass magnetization versus magnetic field curve measurements; temperature dependence of the coercive field measurements; zero field cooling (ZFC) and field cooling (FC) magnetization measurements) were studied using a MPMS- 
XL superconducting quantum interference device (SQUID) from Quantum Design, Inc. The mass magnetization (or specific magnetization) versus magnetic field measurements were performed up to $1,600 \mathrm{kA} / \mathrm{m}$. The $\mathrm{ZFC} / \mathrm{FC}$ measurement procedure was carried out as follows: first, the sample was cooled down from 300 to $5 \mathrm{~K}$ in zero magnetic field; then, a static magnetic field of 50 Oe was applied, and the magnetization was measured while increasing the temperature up to $300 \mathrm{~K}$; and finally, the sample was cooled down again to $5 \mathrm{~K}$ under the same applied magnetic field (50 Oe), and the magnetization was measured while warming up the sample from 5 to $300 \mathrm{~K}$.

\section{Results and discussion}

Based on the ferrogels formulations, the theoretical iron-oxide NP content should be $6 \mathrm{wt} \%$ (mass to mass stoichiometry $\beta=0.06$ ) in anhydrous samples, expressed in terms of completely oxidized $\gamma-\mathrm{Fe}_{2} \mathrm{O}_{3}$. After subtraction of carbonaceous residues arising from incomplete degradation of PVA (1 wt $\%)$ and bound water, the NP content was determined by thermogravimetric analysis and reported in Gonzalez et al. (2011) to be nearly identical to the calculated value for both types of samples, indicating the absence of exudation of NPs during drying. The density of the obtained ferrogels were 1.3 and $1.0 \mathrm{~g} / \mathrm{cm}^{3}$ for the FT and GA samples, respectively. The PVA crystallinity degree and melting temperature showed a slight decrease in the presence of NPs, indicating the formation of less crystallites of smaller size when compared with NP-free PVA matrices (Gonzalez et al. 2011). The GA samples (neat matrix and ferrogel) showed a constant swelling behavior, indicating that the NPs have a small influence on the network properties (Gonzalez et al. 2011). A completely different behavior, however, was observed for the FT samples. In this case, the presence of NPs strongly decreased the swelling capacity of the gel and increased its dimensional stability [a discussion on these effects was presented by Gonzalez et al. (2011)]. In this same work, the size of the NPs was estimated to be $9-10 \mathrm{~nm}$ from XRD and VSM analyses. For our current work, transmission electron microscopy measurements have been used to confirm the NPs size (see Supplementary Information document). The obtained mean diameter values (and the standard deviation indicated in brackets) of the volume-weighted distributions are $7.9 \mathrm{~nm}(0.9 \mathrm{~nm})$ and $8.5 \mathrm{~nm}(1.9 \mathrm{~nm})$ for the FT and GA samples, respectively.

The presence of $\gamma-\mathrm{Fe}_{2} \mathrm{O}_{3}$ NPs in both samples was confirmed through XANES and Mössbauer measurements. Figure 1 shows the Fe $K$-edge XANES spectra for the synthesized ferrogels. The measured spectra of $\gamma-\mathrm{Fe}_{2} \mathrm{O}_{3}$ (powder) and $\mathrm{Fe}_{3} \mathrm{O}_{4}$ (powder) are also included here for comparison purposes. Since the line shape and peak position of the XANES spectra depend on the local electronic structure of the metal ions, they contain information on the oxidation state of the probe ions. The observed XANES spectral features of the ferrogels (GA and FT) were different from those of the reference $\mathrm{Fe}_{3} \mathrm{O}_{4}$ sample, but bore a close resemblance to the $\gamma-\mathrm{Fe}_{2} \mathrm{O}_{3}$ spectrum. The edge position of both samples, as well as of the $\gamma-\mathrm{Fe}_{2} \mathrm{O}_{3}$ reference sample, was around 7,126 eV. However, it is important to notice that the ferrogel peak positions were slightly shifted toward lower energy (around $0.8 \mathrm{eV}$ ) when compared to the $\gamma-\mathrm{Fe}_{2} \mathrm{O}_{3}$ reference. This might be due to the finite-size effects or to the surface contributions.

The ${ }^{57} \mathrm{Fe}$ Mössbauer spectra are sensitive to the local environment of the iron atoms. The hyperfine parameters - the isomer shift $(\delta)$, the quadrupole shift $(\epsilon)$, and the hyperfine magnetic field $(H)$-provide information about the electronic density and its symmetry, and also about the magnetic field experienced by the probe nucleus. The Mössbauer parameters allow the identification of nonequivalent iron positions in a crystal lattice and also the identification

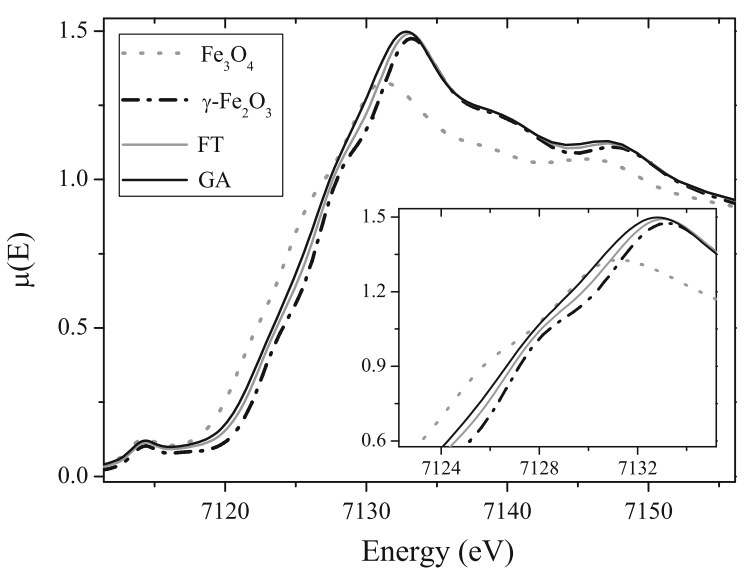

Fig. 1 X-ray absorption near edge spectra (XANES). Inset shows peak positions in more detail 
of compounds and phases (Vandenberghe et al. 2000; Zboril et al. 2002). The Mössbauer spectra of the GA and FT samples obtained are shown in Fig. 2. Qualitatively, the observed spectra were similar to those observed for the iron-oxide magnetic NPs (Zboril et al. 2002).

In the absence of an applied magnetic field, the magnetization direction of a large single-domain particle is along an easy direction. However, since the anisotropy energy decreases as the particle size decreases (Luis et al. 2002), the thermal energy may become comparable to the anisotropy energy of a small particle. In such a case, its magnetic moment may fluctuate during the measurement process. Fluctuations can be divided into collective magnetic excitations (small amplitude fluctuation around an easy direction) and superparamagnetic relaxations (magnetization direction fluctuation among the easy directions) (Morup and Topsoe 1976; Morup et al. 1980). In a particle with uniaxial anisotropy, the simplest form of its total magnetic anisotropy energy is given by:

$$
E(\theta)=K V \sin ^{2} \theta
$$

where $K$ is the effective anisotropy energy constant, $V$ is the volume of the particle, and $\theta$ is the angle between the magnetization direction and the magnetization easy direction. When the superparamagnetic relaxation time (the average time the magnetic moment remains along one easy direction between consecutive jumps) is long compared to the observation time, the magnetization vector remains close to one of the easy directions during the observation time.

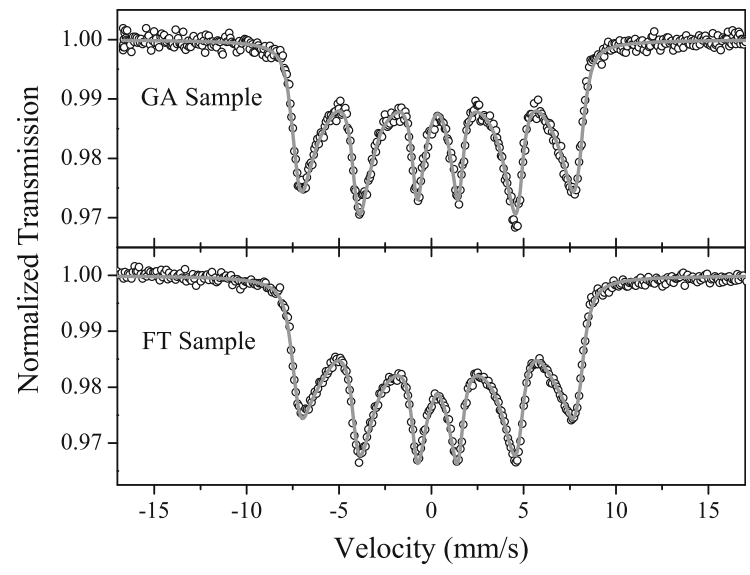

Fig. 2 Mössbauer Spectra of GA and FT samples at $300 \mathrm{~K}$
If the correlation time of the collective magnetic excitations is short compared to the Mössbauer observation time (the nuclear Larmor precession time), the magnetic splitting of the Mössbauer spectrum is proportional to the average value of the hyperfine field:

$H_{\mathrm{hf}}(V, T)=H_{\mathrm{hf}}(V=\infty, T)<\cos \theta>_{T}$

where $H_{\mathrm{hf}}(V=\infty, T)$ is the hyperfine field in a large crystal at $T$ temperature (i.e., in the absence of collective magnetic excitations), and $\langle\cos \theta\rangle_{T}$ is the thermal average of $\cos \theta$ near one of the anisotropy energy minima. The thermal average can be calculated using the partition function of the canonical ensemble (Boltzmann statistics). Hence, the magnetic splitting in the low temperature limit $(K V / k T \gg 1)$ can be written as:

$H_{\mathrm{hf}}(V, T) \simeq H_{\mathrm{hf}}(V=\infty, T)\left(1-\frac{k_{\mathrm{B}} T}{2 K V}\right)$

Thus, the magnetic splitting of a NP Mössbauer spectrum is generally smaller than that of a macroscopic crystal, and depends on the particle volume. If a sample contains NPs with size distribution, the magnetic splitting in particles of different volumes will be different. This results in a broadening of the Mössbauer lines, from which the distribution of $K V$ can be estimated. The broad Mössbauer lines can be seen in the spectra shown in Fig. 2.

The spectra have been fitted with a distribution (histogram) of hyperfine fields resulting from the particle size distribution. The hyperfine parameters at $295 \mathrm{~K}, \delta_{\mathrm{Fe}}$ (isomer shift relative to metallic iron) $=$ $0.340 \pm 0.004 \mathrm{~mm} / \mathrm{s}$ and $\epsilon=0.01 \pm 0.01 \mathrm{~mm} / \mathrm{s}$ for both samples, are consistent with the fact that the NPs have the maghemite structure (Zboril et al. 2002). The obtained hyperfine field distribution reaches its maximum at 46.4 $T$ and $46.3 T$ for the GA and FT samples, respectively. Following the obtained hyperfine field distributions, Eq. 3 (with $H_{\mathrm{hf}}(V=\infty, T)=49.9 T$ [Zboril et al. 2002)], and the $K$ value obtained from the magnetic measurements introduced below, it is possible to determine the NP size distributions. The mean diameters (and the standard deviation indicated in brackets) obtained are $9.0 \mathrm{~nm}(1.4 \mathrm{~nm})$ and $9.3 \mathrm{~nm}$ $(1.5 \mathrm{~nm})$ for the GA and FT samples, respectively.

Coercive fields were measured for several temperatures and plotted in Fig. 3. In general, the coercive 
field behavior of random-oriented and non-interacting systems follows equation:

$H_{\mathrm{c}}(T)=0.48 \frac{2 K}{\mu_{0} M_{\mathrm{s}} \rho}\left[1-\left(\frac{T}{\left\langle T_{\mathrm{B}}\right\rangle}\right)^{\frac{1}{2}}\right]$

where $K$ is the effective anisotropy constant, $M_{\mathrm{s}}$ is the saturation magnetization, $\rho$ is the sample density, and $\left\langle T_{\mathrm{B}}\right\rangle$ is the blocking temperature meanvalue. Figure 3 shows that both samples closely follow this dependence. The small departure of $H_{\mathrm{c}}$ $\left(T^{1 / 2}\right)$ from linearity may be due to an effect of the NP size distribution or to a weak NP dipolar interaction. The associated NP moment distribution gives rise to a blocking temperature distribution. This behavior was well described by Nunes et al. (2004). Above $200 \mathrm{~K}, H_{\mathrm{c}}$ does not experience any important changes, which may indicate the presence of a small fraction of unusually large particles or the existence of a segregated and magnetically ordered macrophase. Inset in Fig. 3 shows the coercive field as a function of $T^{1 / 2}$ ranging from 5 to $85 \mathrm{~K}$. By extrapolating the linear fit to $H_{\mathrm{c}}=0$, we have determined the mean blocking temperature which results in $\left\langle T_{\mathrm{B}}\right\rangle=84 \pm$ $9 \mathrm{~K}$ for the FT sample, and in $\left\langle T_{\mathrm{B}}\right\rangle=89 \pm 9 \mathrm{~K}$ for the GA sample. Using Eq. 4, the extrapolation of $H_{\mathrm{c}}(T)$ to $T=0$ allows us to determine both samples anisotropy constants $(K): K^{\mathrm{FT}}=13.0 \pm 0.3 \mathrm{~kJ} / \mathrm{m}^{3}$ for the FT sample, and $K^{\mathrm{GA}}=13.9 \pm 0.3 \mathrm{~kJ} / \mathrm{m}^{3}$ for the $\mathrm{GA}$ sample.

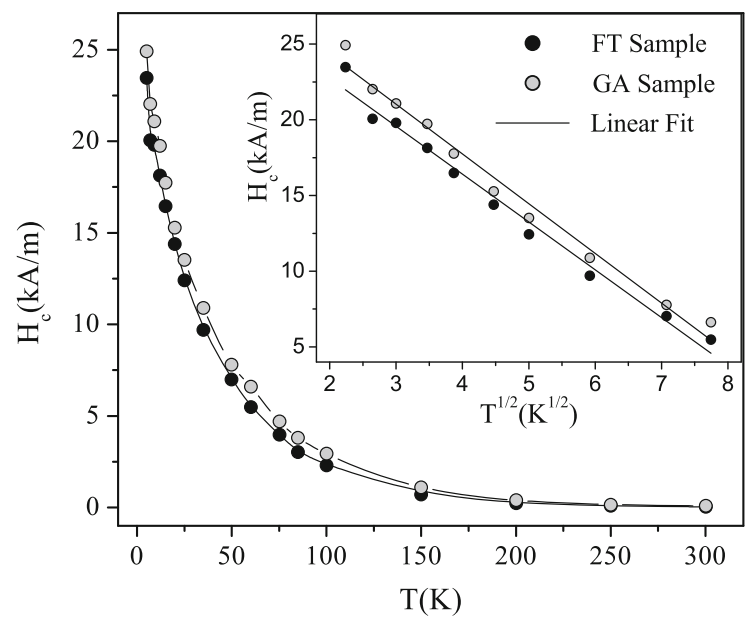

Fig. 3 Temperature dependence of the coercivity $H_{\mathrm{c}}$ for FT and GA samples measured using a SQUID magnetometer. The inset indicates that the coercive field follows the $T^{1 / 2}$ dependence
Figure 4 shows the temperature dependence of the ZFC and FC magnetizations in the range of 5 to $300 \mathrm{~K}$ for both samples. Following the ZFC and FC curves, one can determine the mean blocking temperature (defined as the $d(\mathrm{FC}-\mathrm{ZFC}) / d T$ maximum value) (Micha et al. 2004) at about 107 and $110 \mathrm{~K}$ for the FT and GA samples, respectively. These values are close to those obtained from the measurement of the coercive field temperature dependence. So far, we have not considered any kind of interparticle interactions, but the almost constancy of $M(T)$ below the maximum ZFC magnetization value suggests the existence of dipolar interactions. This may lead to an increase of $T_{\mathrm{B}}$ in comparison with the blocking temperature of non-interacting NP samples. This phenomenon has been discussed in previous works (Hoppe et al. 2008; Serantes et al. 2010b). In fact, based on the mean size of the iron-oxide NPs, lower blocking temperatures than the observed ones would be expected in the absence of interactions.

The $M$ versus $H$ curves were measured at 5, 50, 100, 150, 200, 250, and $300 \mathrm{~K}$. Figures 5 and 6 show curves measured at four different temperatures $(5,50,150$, and $300 \mathrm{~K})$ for the FT and GA samples, respectively. Considering the gels maghemite concentration $(6 \mathrm{wt} \%)$ and the observed saturation magnetization values of $\sim 3.6$ and $\sim 3.4 \mathrm{emu} / \mathrm{g}$ for the FT and GA samples, respectively, the saturation magnetization of NPs at $300 \mathrm{~K}$ can be calculated to be $\sim 60.6$ and $\sim 56.4 \mathrm{emu} / \mathrm{g}$, respectively. As it has already been said, it is well known that saturation magnetization of iron-oxide NPs depends on their size

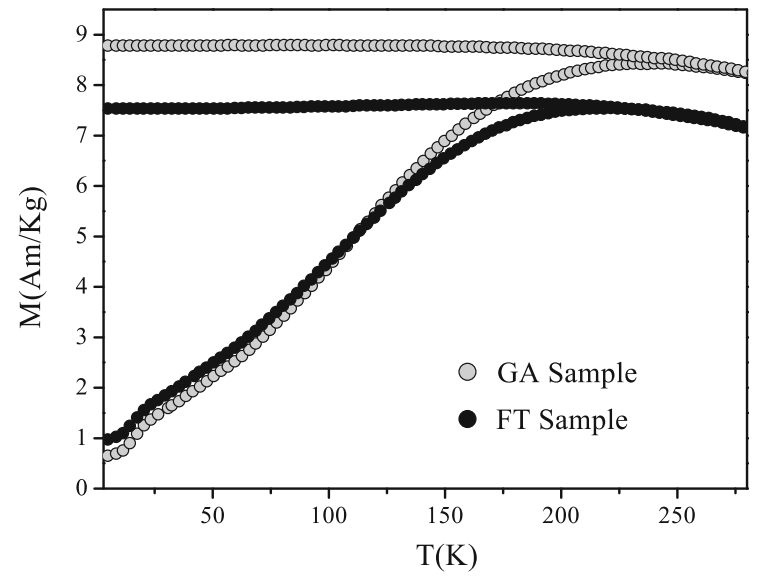

Fig. 4 Temperature dependence of the ZFC and FC magnetizations for FT and GA samples 
(collective excitations) and on their surface magnetic configuration (disorder). These phenomena can reduce the saturation magnetization below the bulk expected value (Lima et al. 2010; Laurent et al. 2008; Wu et al. 2007). One example of this effect has been published in a recent work by Laurent et al. (2008). They report room temperature (RT) saturation magnetization values of 20 and $58 \mathrm{emu} / \mathrm{g}$ for iron-oxide NPs with average sizes of 4.48 and $5.59 \mathrm{~nm}$, respectively. $\mathrm{Wu}$ et al. (2007) have reported saturation magnetization values between 16.80 and $24.10 \mathrm{emu} / \mathrm{g}$ for magnetite NPs with sizes below $5 \mathrm{~nm}$; Vargas et al. (2008) have obtained saturation magnetization values of approximately $20 \mathrm{emu} / \mathrm{g}$ for $5 \mathrm{~nm}$ magnetite particles; Goya et al. (2003) have reported values between 55 and $65 \mathrm{emu} / \mathrm{g}$ for $20 \mathrm{~nm}$ magnetite NPs at RT. Hence, the saturation magnetization results obtained for the GA and FT samples in this work are consistent with the values presented above.

For illustration purposes, the bottom right insets of Figs. 5 and 6 show the coercive field and the remanence zone of the $M$ versus $H$ curves. The unblocked (superparamagnetic) regime can be observed for temperatures above $200 \mathrm{~K}$.

To better understand the obtained $M$ versus $H$ curves, a Langevin fitting was performed. When the magnetic material is composed of monodispersed non-interacting particles, the superparamagnetic contribution to the total mass magnetization can be described by $M=M_{\mathrm{s}} L(x)$, where $L(x)$ is the Langevin

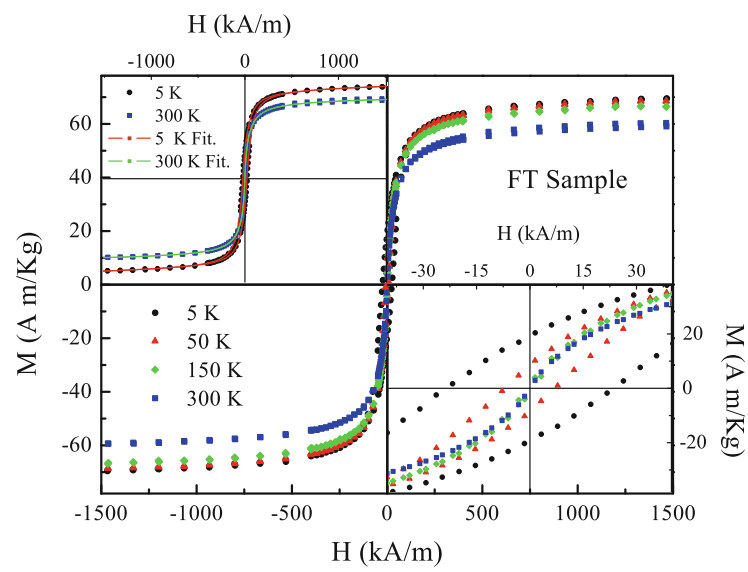

Fig. $5 M$ versus $H$ curves for FT sample at 5, 50, 150, and $300 \mathrm{~K}$. The top left inset shows the data and the corresponding fitting curves for the 5 and $300 \mathrm{~K}$ measurements. The bottom right inset is a zoom-in of the low field region showing the coercivity behavior at different temperatures

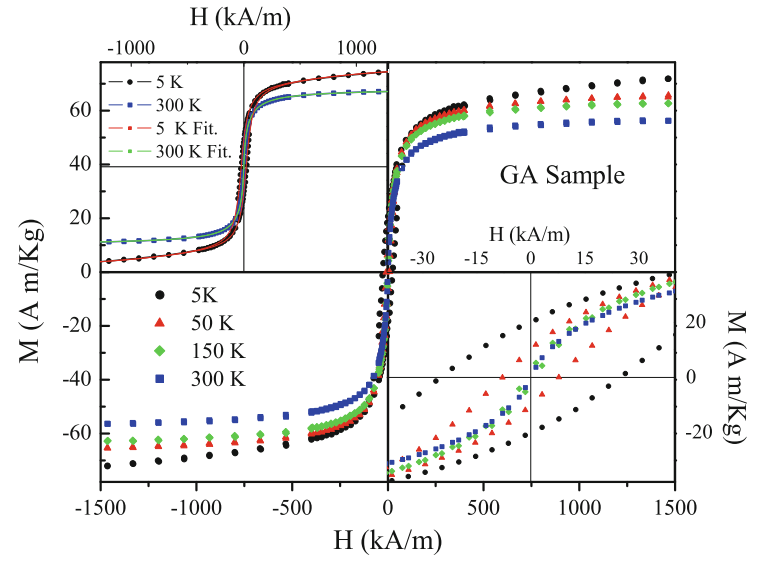

Fig. $6 M$ versus $H$ curves for GA sample at 5, 50, 150, and $300 \mathrm{~K}$. The top left inset shows the data and the corresponding fitting curves for the 5 and $300 \mathrm{~K}$ measurements. The bottom right inset is a zoom-in of the low field region showing the coercivity behavior at different temperatures

function and $x=\mu_{0} \mu H / k_{\mathrm{B}} T ; \mu_{0}$ being the vacuum permeability; $\mu$, the particle magnetic moment; and $M_{\mathrm{s}}=\mu N$, the saturation magnetization of the $N$ particles per mass of maghemite in the samples. In our case, a more detailed description is needed due to the presence of particle size distributions containing partially-blocked large particles $(L)$ and unblocked small particles $(S)$. Considering that the coercivity values of $L$ particles are small in comparison with the anisotropy field, these particles have moment states which are not too far from the equilibrium state.

Under our model, mass magnetization of the $S$ particles, $M^{\mathrm{S}}(T)$, is described by equation:

$M^{S}=M_{\mathrm{s}}^{S} L\left(\mu_{m}^{S}\right)$

where $\mu_{m}^{\mathrm{S}}$ and $M_{\mathrm{s}}^{\mathrm{S}}$ are the mean magnetic moment and saturation mass magnetization of the unblocked particles, respectively. As regards $L$ particles, and considering that the magnetic moment of the particles is not far from the equilibrium state, we assume that a modified Langevin function $L\left(x-x_{\mathrm{c}}\right)$, where $x_{\mathrm{c}}=\mu_{0} \mu H_{\mathrm{c}} / k_{\mathrm{B}} T$, can be used. Here, the argument of the Langevin function has been modified to be able to describe the appearing coercivity below $200 \mathrm{~K}$.

Since the samples present polydispersity, a lognormal size distribution is introduced (Ferrari et al. 1997; Cezar et al. 2001). Therefore, the mass magnetization contribution of the $L$ particle fraction, $M^{L}(T)$, is described by: 
$M^{L}=\int_{0}^{\infty} \mu^{L} L\left(\frac{\mu_{0} \mu^{L}\left(H-H_{c}\right)}{k_{\mathrm{B}} T}\right) f\left(\mu^{L}\right) d \mu^{L}$

where $f(\mu)$ is the magnetic moment number-distribution function. Mass saturation magnetization of the $L$ particles is defined by equation:

$M_{S}^{L}=\int_{0}^{\infty} \mu^{L} f\left(\mu^{L}\right) d \mu^{L}=N_{P}^{L} \mu_{m}^{L}$

where $N_{P}^{L}$ is the total number of particles per mass unit, and $\mu_{m}^{L}$ is the mean magnetic moment of the $L$ particles. Calculations were performed using a log-normal distribution of $\mu$ values, defined as:

$f(\mu)=\frac{N_{P}}{\sqrt{2 \pi} \mu \sigma} \exp \left(-\frac{\ln ^{2}\left(\mu / x_{0}\right)}{2 \sigma^{2}}\right)$

where $\sigma$ is the standard deviation and $x_{0}$ is the median of the distribution. This median value is related to the mean magnetic moment $\mu_{m}^{L}$ by $\mu_{m}^{L}=x_{0} e^{\sigma^{2} / 2}$.

The $M$ versus $H$ curves obtained within a $5-300 \mathrm{~K}$ range were fitted using the model described above. The top left insets of Figs. 5 and 6 show the experimental magnetization loops for two temperatures ( 5 and $300 \mathrm{~K}$ ), along with the fitted curves. This confirms that our model is in agreement with the experimental data of both samples at different temperatures. Table 1 summarizes the best-fitting parameters for measurements at all studied temperatures.

Table 1 shows the decrease of saturation magnetization $M_{S}^{T}$ with temperature, as observed in our experiments. However, $\mu_{m}^{L}$ values show a non-physical increase with temperature. The expected behavior of $\mu_{m}^{L}$ is a slow decrease at low temperatures, and a faster decrease when approaching the Curie temperature. The particles moment unrealistic behavior was reported by Allia et al. (2001) for a system where dipolar magnetic interactions among particles cannot be neglected. They suggested that this phenomenon can be explained on the basis of the collective magnetic interactions, since the magnetic moments of NPs interact with each other through long-range dipolar random forces. This phenomenon can be pictured in terms of a temperature-like quantity, $T^{*}$, which must be added to temperature $T$ in the Langevin function argument. This is justified because the random nature of the interparticle dipolar field produces a random effect on the direction of the magnetic moments. This random effect disorders the magnetic moments and opposes the ordering effect of the external magnetic field. Thus, the NP dipolar interactions can be considered as an increase source of the effective temperature. This extra temperature, $T^{*}$, is related to the dipolar energy $\varepsilon_{D}$ through:

Table 1 Best-fitting parameter values obtained for $M$ versus $H$ experimental curves for both FT and GA samples, where $T$ is the temperature in $\mathrm{K} ; x_{0}, \mu_{m}^{L}, \mu^{S}$ and $\mu_{\mathrm{Fe}}$ are in $\mu_{\mathrm{B}} ; M_{\mathrm{s}}^{L}, M_{\mathrm{s}}^{S}$ and $M_{\mathrm{s}}^{T}$ are in $\mathrm{Am}{ }^{2} / \mathrm{Kg}$; and $H_{\mathrm{c}}$ is in $\mathrm{kA} / \mathrm{m}$

\begin{tabular}{lrlrlrlrrr}
\hline & \multicolumn{1}{c}{$T$} & $\mu_{m}^{S}$ & \multicolumn{1}{c}{$M_{\mathrm{s}}^{S}$} & \multicolumn{1}{c}{$x_{0}$} & \multicolumn{1}{c}{$\mu_{m}^{L}$} & \multicolumn{1}{c}{$M_{\mathrm{s}}^{L}$} & \multicolumn{1}{c}{$H_{\mathrm{c}}$} & $M_{\mathrm{s}}^{T}$ & $\mu_{\mathrm{Fe}}$ \\
\hline FT & 5 & $110(3)$ & $27.9(0.7)$ & $353(2)$ & $352(2)$ & $42.2(0.3)$ & $27.7(0.4)$ & $70(1)$ & $0.97(0.01)$ \\
FT & 50 & $322(12)$ & $8.0(0.3)$ & $2369(29)$ & $2688(57)$ & $62.4(1.3)$ & $6.1(0.1)$ & $70(1)$ & $0.97(0.02)$ \\
FT & 100 & $344(10)$ & $4.8(0.1)$ & $3167(19)$ & $4363(47)$ & $65.3(0.7)$ & $1.86(0.06)$ & $70.1(0.8)$ & $0.97(0.01)$ \\
FT & 150 & $343(9)$ & $3.9(0.1)$ & $3557(16)$ & $5648(45)$ & $65.5(0.5)$ & $0.51(0.05)$ & $69.4(0.6)$ & $0.96(0.01)$ \\
FT & 200 & $369(21)$ & $2.6(0.2)$ & $3727(23)$ & $6616(75)$ & $65.1(0.7)$ & $0.15(0.06)$ & $67.8(0.9)$ & $0.94(0.01)$ \\
FT & 250 & $347(12)$ & $3.2(0.1)$ & $4423(19)$ & $8112(63)$ & $62.1(0.5)$ & $0(0.05)$ & $65.2(0.6)$ & $0.90(0.01)$ \\
FT & 300 & $599(35)$ & $2.8(0.2)$ & $4992(39)$ & $9400(123)$ & $59.3(0.8)$ & $0(0.07)$ & $62(1)$ & $0.86(0.01)$ \\
GA & 5 & $10.3(0.3)$ & $17.1(0.5)$ & $217(3)$ & $234(6)$ & $62(1.6)$ & $23.2(0.3)$ & $79(2)$ & $1.10(0.03)$ \\
GA & 50 & $260(9)$ & $8.0(0.5)$ & $2399(31)$ & $2845(64)$ & $59(1.3)$ & $7.0(0.1)$ & $67(2)$ & $0.93(0.02)$ \\
GA & 100 & $356(12)$ & $4.7(0.2)$ & $3184(24)$ & $4658(64)$ & $61(0.8)$ & $2.48(0.07)$ & $66(1)$ & $0.91(0.01)$ \\
GA & 150 & $349(13)$ & $2.9(0.2)$ & $3164(17)$ & $5629(54)$ & $62(0.6)$ & $0.89(0.05)$ & $65.1(0.7)$ & $0.90(0.01)$ \\
GA & 200 & $327(15)$ & $2.3(0.1)$ & $3185(16)$ & $6512(58)$ & $61(0.6)$ & $0.25(0.04)$ & $63.6(0.7)$ & $0.88(0.01)$ \\
GA & 250 & $378(19)$ & $2.0(0.1)$ & $3374(18)$ & $7477(74)$ & $60(0.6)$ & $0(0.05)$ & $61.7(0.7)$ & $0.85(0.01)$ \\
GA & 300 & $350(34)$ & $1.4(0.5)$ & $3683(21)$ & $8472(87)$ & $57(0.6)$ & $0(0.05)$ & $58.7(0.7)$ & $0.81(0.01)$ \\
\hline
\end{tabular}

The calculated values for $M_{\mathrm{s}}^{T}=M_{\mathrm{s}}^{S}+M_{\mathrm{s}}^{L}$ and the mean magnetic moment per Fe atom $\left(\mu_{\mathrm{Fe}}\right)$ are also included 
$k_{B} T^{*}=\varepsilon_{D}$

where $\varepsilon_{D}=\alpha \mu^{2} / d^{3}, \mu$ being the NP true magnetic moment; $d$, the average interparticle distance; and $\alpha$, a proportionality constant derived from the sum of all dipolar energy contributions. The validity of this assumption has been recently confirmed by Allia and Tiberto (2011).

Following Allia et al. (2001), the mean and meanquadratic values of the apparent NP moment can be related to other relevant quantities of the model by means of the equation:

$\frac{\xi}{\chi}=\frac{3 k_{B} N_{P}}{\mu_{0}}\left(\frac{T}{M_{S}^{2}}\right)+3 \beta \frac{\alpha}{\mu_{0}}$.

where $\xi=\left\langle\mu^{2}>/<\mu\right\rangle^{2}(<\mu>$ being the average value of the NPs' magnetic moment; and $<\mu^{2}>$, the average square value of the NPs' magnetic moment), $\chi$ is the low field mass susceptibility, and $\beta$ is the mass density of NPs in the ferrogel. Here, $\xi$ has the same value for the distribution of both, the apparent and the real, magnetic moments. In order to obtain the $T^{*}$ values and the true mean magnetic moment $\mu$, we fitted experimental quantity $\xi / \chi$ against $T / M_{S}^{2}$ using Eq. 10.

A linear dependence of quantity $\xi / \chi$ on $T / M_{S}^{2}$ has been experimentally observed for the FT and GA samples within the studied temperature range, with the exception of the data obtained at $5 \mathrm{~K}$, as can be seen in Fig. 7. The marked deviation from linearity at $5 \mathrm{~K}$ is probably related to the single-particle moment blocking. At this temperature, the $M$ versus $H$ curves for both samples reveal a coercive field higher than $20 \mathrm{kA} / \mathrm{m}$.

The fitted values of the number of NPs per ferrogel mass unit are $N_{P}=4.4 \times 10^{19} \mathrm{Kg}^{-1}$ and $N_{P}=$ $3.1 \times 10^{19} \mathrm{Kg}^{-1}$ for the FT and GA samples, respectively. These values correspond to $\mu=9500 \mu_{\mathrm{B}}$, $\alpha=5.66 \times 10^{-6}, T^{*}=99 \mathrm{~K}$, and to $\varepsilon_{D}=1.37 \times$ $10^{-21} \mathrm{~J}$ for the FT sample; and $\mu=12,600 \mu_{\mathrm{B}}$, $\alpha=3.03 \times 10^{-6}, T^{*}=173 \mathrm{~K}$, and to $\varepsilon_{D}=2.38 \times$ $10^{-21} \mathrm{~J}$ for the GA sample (all values at $300 \mathrm{~K}$ ). The obtained mean magnetic moments correspond to mean diameters of 8.1 and $9.1 \mathrm{~nm}$ for the FT and GA samples, respectively. The average interparticle distance $d$ could also be obtained: 26 and $32 \mathrm{~nm}$ for the FT and GA samples, respectively. The obtained particle size values are in agreement with those

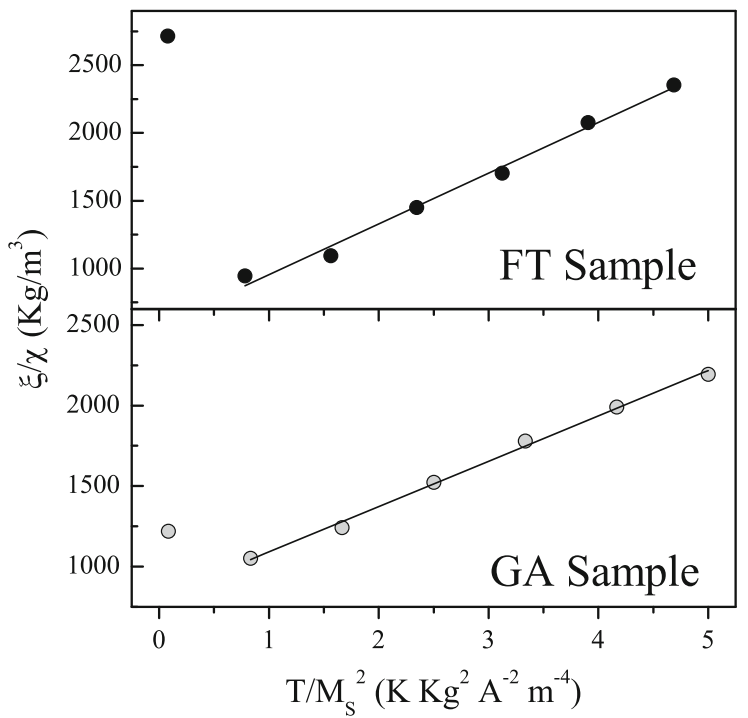

Fig. 7 Experimental plot of $\frac{\xi}{\chi}$ versus $T / M_{S}^{2}$ for GA and FT samples. The lines correspond to the best fits using Eq. 10

obtained by means of the Mössbauer spectroscopy. It is interesting to notice that while the mean interparticle distance is smaller in the FT sample than in the GA sample, the dipolar interaction energy is also smaller in the FT ferrogels. The fact that the dipolar interaction energy is smaller in the FT sample is in agreement with the fact that the magnetic moment of NPs is also smaller. Also, the smaller mean interparticle distance in the FT sample is in agreement with the higher value of $\alpha$, since $\alpha$ is largely influenced by the distances among the nearest NPs. Within this frame of reference, we may assume that the GA sample has a higher degree of inhomogeneity, such as a higher tendency to form NP aggregates, for example.

\section{Conclusions}

In this work, we have demonstrated that the FT and GA samples show very similar magnetic properties. Through XANES and Mössbauer measurements, the iron-oxide phase present in both samples was identified as $\gamma-\mathrm{Fe}_{2} \mathrm{O}_{3}$. Mössbauer measurements show that the NP magnetic moments present collective magnetic excitations and superparamagnetic relaxations. The FT and GA samples do not show significant differences in regard to other relevant properties either: taking into account the experimental uncertainties, 
both samples have the same values for effective anisotropy constant $\left(\sim 14 \mathrm{~kJ} / \mathrm{m}^{3}\right)$, mean NP size $(\sim 9 \mathrm{~nm})$, and mean hyperfine field $(\sim 46 T)$, while the saturation magnetization values are close to each other ( $\sim 61$ and $\sim 56 \mathrm{emu} / \mathrm{g}$ for the FT and GA samples, respectively). ZFC - FC measurements have allowed us to confirm the superparamagnetic state of both materials at room temperature. In view of these similarities, the performance of both types of ferrogels should be similar for most applications. There are, however, two important differences in relation to the preparation and potential medical applications of these materials: the FT ferrogels are easier to prepare than the GA ferrogels, and they also leave no toxic impurities in the final product.

Having analyzed the magnetization values as a function of the applied fields at different temperatures, and having described those measurements on the basis of a Langevin-like response distribution while differentiating between the blocked and unblocked particle contributions, we have obtained the NP main parameters, determining the magnetic moment distribution of the samples and their temperature dependence. From this analysis, we have observed that the temperature dependence of the mean magnetic moment presents a non-physical behavior. On the basis of a model proposed by Allia and Tiberto (2011), a meaningful real temperature dependence of the magnetic moment was recovered by taking into account the magnetic dipolar interactions among NPs. Based on this model, it was possible to determine other important parameters like the average distance between magnetic NPs ( $26 \mathrm{~nm}$ for FT and $32 \mathrm{~nm}$ for GA), the mean number of NPs per ferrogel mass $\left(4.4 \times 10^{19}\right.$ and $3.1 \times 10^{19} \mathrm{Kg}^{-1}$ for FT and GA), and the intensity of the interparticle dipolar energy $\left(1.37 \times 10^{-21}\right.$ and $2.38 \times 10^{-21} \mathrm{~J}$ for FT and GA $)$. The larger dipolar energy observed for the GA sample has been attributed to a less homogeneous distribution of NPs, which would give rise to small regions with higher NP concentration.

Acknowledgements The work at UNICAMP was supported by FAPESP and CNPq, Brazil. We appreciate the financial support of LNLS, Campinas, Sao Paulo, Brazil (Research Project D04B-XAFS1-10818 - XAS Study of Nanomaterials for Biomedical Applications); CONICET, Argentina (PIP 01111); and ANPCyT, Argentina (PICT 00898, PICT 2010-2721). The authors also want to thank C. Hoppe for her helpful comments and suggestions.

\section{References}

Allia P, Coisson M, Tiberto P, Vinai F, Knobel M, Novak MA, Nunes WC (2001) Granular $\mathrm{Cu}-\mathrm{Co}$ alloys as interacting superparamagnets. Phys Rev B 64:144420. doi:10.1103/ PhysRevB.64.144420

Allia P, Tiberto P (2011) Dynamic effects of dipolar interactions on the magnetic behavior of magnetite nanoparticles. J Nanopart Res 13:7277-7293 doi:10.1007/s11051011-0642-2

Bray JC, Merrill EW (1973) Poly(vinyl alcohol) hydrogels. Formation by electron beam irradiation of aqueous solutions and subsequent crystallization. J Appl Polym Sci 17(12):3779-3794. doi:10.1002/app.1973.070171219

Cezar JC, Knobel M, Tolentino HC (2001) Magnetic properties of $\mathrm{Cu}$-permalloy granular alloy. J Magn Magn Mater 226(0): 1519-1521, doi:10.1016/S0304-8853(00)00944-6

Chandrasekar M, Suresh S, Senthilkumar T (2012) Mechanisms proposed through experimental investigations on thermophysical properties and forced convective heat transfer characteristics of various nanofluids-a review. Renew Sustain Energy Rev 16(6):3917-3938, doi:10.1016/j.rser. 2012.03.013

Chen J, Yang L, Liu Y, Ding G, Pei Y, Li J, Hua G, Huang J (2005) Preparation and characterization of magnetic targeted drug controlled-release hydrogel microspheres. Macromol Symp 225(1):71-80 doi:10.1002/masy.20055 0706

Cima LG, Lopina ST (1995) Network structures of radiationcrosslinked star polymer gels. Macromolecules 28(20): 6787-6794 doi:10.1021/ma00124a013

Faidley L, Han Y, Tucker K, Timmons S, Hong Wc (2010) Axial strain of ferrogels under cyclic magnetic fields. Smart Mater Struct 19(7). doi:10.1088/0964-1726/19/ 7/075001

Ferrari EF, da Silva FCS, Knobel M (1997) Influence of the distribution of magnetic moments on the magnetization and magnetoresistance in granular alloys. Phys Rev B 56:6086-6093. doi:10.1103/PhysRevB.56.6086

François NJ, Allo S, Jacobo SE, Daraio ME (2007) Composites of polymeric gels and magnetic nanoparticles: preparation and drug release behavior. J Appl Polym Sci 105(2): 647-655. doi:10.1002/app.26321

Furukawa H, Shimojyo R, Ohnishi N, Fukuda H, Kondo A (2003) Affinity selection of target cells from cell surface displayed libraries: a novel procedure using thermoresponsive magnetic nanoparticles. Appl Microbiol Biotechnol 62:478-483. doi:10.1007/s00253-003-1330-7

Gonzalez J, Hoppe C, Muraca D, Sánchez F, Alvarez V (2011) Synthesis and characterization of PVA ferrogels obtained through a one-pot freezing-thawing procedure. Colloid Polym Sci 289:1839-1846. doi:10.1007/s00396-011-2501-1

Gonzalez JS, Hoppe CE, Alvarez VA (2012) Advances in materials science research. In: Poly (vinyl alcohol) ferrogels: synthesis and applications, Chapter 8, Nova Science Publishers, Inc., New York

Goya GF, Berquó TS, Fonseca FC, Morales MP (2003) Static and dynamic magnetic properties of spherical magnetite nanoparticles. J Appl Phys 94(5):3520-3528. doi:10.1063/ 1.1599959 
Hassan C, Peppas N (2000) Structure and applications of poly(vinyl alcohol) hydrogels produced by conventional crosslinking or by freezing/thawing methods. In: Biopolymers PVA hydrogels, anionic polymerisation nanocomposites, Adv. Polym. Sci., vol 153. Springer Heidelberg, pp 37-65, doi:10.1007/3-540-46414-X_2

Hill DJT, Whittaker AK, Zainuddin (2011) Water diffusion into radiation crosslinked PVA-PVP network hydrogels. Radiat Phys Chem 80(2):213-218. doi:10.1016/j.radphys chem.2010.07.035

Hoppe CE, Rivadulla F, Arturo López-Quintela M, Carmen Bujan M, Rivas J, Serantes D, Baldomir D (2008) Effect of submicrometer clustering on the magnetic properties of free-standing superparamagnetic nanocomposites. J Phys Chem C 112(34):13099-13104. doi:10.1021/jp8039548

Jolivet JP, Chanéac C, Tronc E (2004) Iron oxide chemistry. From molecular clusters to extended solid networks. Chem Commun 0:481-483. doi:10.1039/B304532N

Kim YI, Kim D, Lee CS (2003) Synthesis and characterization of $\mathrm{CoFe}_{2} \mathrm{O}_{4}$ magnetic nanoparticles prepared by temperature-controlled coprecipitation method. Physica B 337(14): 42-51. doi:10.1016/S0921-4526(03)00322-3

Knobel M, Nunes W, Socolovsky L, De Biasi E, Vargas J, Denardin J (2008) Superparamagnetism and other magnetic features in granular materials: a review on ideal and real systems. J Nanosci Nanotechnol 8(6):2836-2857. http:// www.ingentaconnect.com/content/asp/jnn/2008/00000008/ 00000006/art00009

Krishnan KM (2010) Biomedical nanomagnetics: a spin through possibilities in imaging, diagnostics, and therapy. IEEE Trans Magn 46(7):2523-2558. doi:10.1109/TMAG.2010. 2046907

Laurent S, Forge D, Port M, Roch A, Robic C, Vander Elst L, Muller RN (2008) Magnetic iron oxide nanoparticles: synthesis, stabilization, vectorization, physicochemical characterizations, and biological applications. Chem Rev 108(6):2064-2110. doi:10.1021/cr068445e

Liang X, Wang X, Zhuang J, Chen Y, Wang D, Li Y (2006) Synthesis of nearly monodisperse iron oxide and oxyhydroxide nanocrystals. Adv Funct Mater 16(14):1805-1813. doi:10.1002/adfm.200500884

Lima E, De Biasi E, Vasquez Mansilla M, Saleta ME, Effenberg F, Rossi LM, Cohen R, Rechenberg HR, Zysler RD (2010) Surface effects in the magnetic properties of crystalline $3 \mathrm{~nm}$ ferrite nanoparticles chemically synthesized. J Appl Phys 108(10):103919. doi:10.1063/1.3514585

Liu TY, Hu SH, Liu TY, Liu DM, Chen SY (2006) Magneticsensitive behavior of intelligent ferrogels for controlled release of drug. Langmuir 22(14):5974-5978. doi:10.1021/la060 $371 \mathrm{e}$

Longo A, Wang X, Ruotolo A, Peluso A, Carotenuto G, Lortz R (2012) Effect of the polymeric matrix on the structural and magnetic properties of hematite/polymer composites. J Nanopart Res 14:1-8. doi:10.1007/s11051-012-1314-6

Luis F, Torres JM, García LM, Bartolomé J, Stankiewicz J, Petroff F, Fettar F, Maurice JL, Vaurés A (2002) Enhancement of the magnetic anisotropy of nanometersized Co clusters: Influence of the surface and of interparticle interactions. Phys Rev B 65:094, 409. doi:10.1103/ PhysRevB.65.094409
Mao L, Hu Y, Piao Y, Chen X, Xian W, Piao D (2005) Structure and character of artificial muscle model constructed from fibrous hydrogel. Curr Appl Phys 5(5):426-428. doi: 10.1016/j.cap.2004.11.003

Masoudi A, Hosseini HRM, Shokrgozar MA, Ahmadi R, Oghabian MA (2012) The effect of poly(ethylene glycol) coating on colloidal stability of superparamagnetic iron oxide nanoparticles as potential MRI contrast agent. Int J Pharm 433(12):129-141 doi:10.1016/j.ijpharm.2012.04.080

Matsuyama H, Teramoto M, Urano H (1997) Analysis of solute diffusion in poly(vinyl alcohol) hydrogel membrane. J Membr Sci 126(1):151-160. doi:10.1016/S0376-7388 (96)00287-6

Mawad D, Odell R, Poole-Warren LA (2009) Network structure and macromolecular drug release from poly(vinyl alcohol) hydrogels fabricated via two crosslinking strategies. Int J Pharm 366(12):31-37. doi:10.1016/j.ijpharm.2008.08.038

Micha J, Dieny B, Régnard J, Jacquot J, Sort J (2004) Estimation of the Co nanoparticles size by magnetic measurements in $\mathrm{Co} / \mathrm{SiO}_{2}$ discontinuous multilayers. J Magn Magn Mater 272276, Supplement(0):E967-E968. doi:10.1016/j.jmmm.2003. 12.268

Mitsumata T, Ikeda K, Gong J, Osada Y, Szabó D, Zrínyi M (1999) Magnetism and compressive modulus of magnetic fluid containing gels. J Appl Phys 85(12):8451-8455. doi: 10.1063/1.370626

Miyata T, Uragami T, Nakamae K (2002) Biomolecule-sensitive hydrogels. Adv Drug Delivery Rev 54(1):79-98. doi: 10.1016/S0169-409X(01)00241-1

Morup S, Topsoe H (1976) Mössbauer studies of thermal excitations in magnetically ordered microcrystals. Appl phys 11:63-66. doi:10.1007/BF00895017

Morup S, Dumesic JA, Topsoe H (1980) Applications of Mössbauer Spectroscopy. Academic Press Inc., New York

Nunes WC, Folly WSD, Sinnecker JP, Novak MA (2004) Temperature dependence of the coercive field in singledomain particle systems. Phys Rev B 70:014, 419. doi: 10.1103/PhysRevB.70.014419

Pankhurst QA, Thanh NKT, Jones SK, Dobson J (2009) Progress in applications of magnetic nanoparticles in biomedicine. J Phys D Appl Phys 42(22). doi:10.1088/00223727/42/22/224001

Peppas NA, Mongia NK (1997) Ultrapure poly(vinyl alcohol) hydrogels with mucoadhesive drug delivery characteristics. Eur J Pharm Biopharm 43(1):51-58. doi:10.1016/ S0939-6411(96)00010-0

Pich A, Bhattacharya S, Lu Y, Boyko V, Adler HJP (2004) Temperature-sensitive hybrid microgels with magnetic properties. Langmuir 20(24):10706-10711. doi:10.1021/la040 $084 \mathrm{f}$

Qiu Y, Park K (2001) Environment-sensitive hydrogels for drug delivery. Adv Drug Deliv Rev 53(3):321-339. doi:10.1016/ S0169-409X(01)00203-4

Raikher Y, Stolbov O (2005) Magnetodeformational effect in ferrogel objects. J Magn Magn Mater 289(0):62-65. doi: 10.1016/j.jmmm.2004.11.018

Rivas J, Bañobre-López M, Piñeyro-Redondo Y, Rivas B, López-Quintela M (2012) Magnetic nanoparticles for application in cancer therapy. J Magn Magn Mater 324(21): 3499-3502. doi:10.1016/j.jmmm.2012.02.075 
Samba Sivudu K, Rhee K (2009) Preparation and characterization of $\mathrm{pH}$-responsive hydrogel magnetite nanocomposite. Colloids Surf A 349(13):29-34. doi:10.1016/j.cols urfa.2009.07.048

Schexnailder P, Schmidt G (2009) Nanocomposite polymer hydrogels. Colloid Polym Sci 287:1-11. doi:10.1007/s00396008-1949-0

Serantes D, Baldomir D, Pereiro M, Botana J, Prida VM, Hernando B, Arias JE, Rivas J (2010a) Magnetocaloric effect in magnetic nanoparticle systems: how to choose the best magnetic material? J Nanosci Nanotechnol 10(4):25122517. doi:10.1166/jnn.2010.1424

Serantes D, Baldomir D, Pereiro M, Hoppe CE, Rivadulla F, Rivas J (2010b) Nonmonotonic evolution of the blocking temperature in dispersions of superparamagnetic nanoparticles. Phys Rev B 82:134433. doi:10.1103/Phys RevB.82.134433

Sholklapper TZ, Jacobson CP, Visco SJ, De Jonghe LC (2008) Synthesis of dispersed and contiguous nanoparticles in solid oxide fuel cell electrodes. Fuel Cells 8(5):303-312. doi:10.1002/fuce. 200800030

Vandenberghe R, Barrero C, da Costa G, Van San E, De Grave E (2000) Mössbauer characterization of iron oxides and (oxy)hydroxides: the present state of the art. Hyperfine Interact 126:247-259. doi:10.1023/A:1012603603203

Vargas J, Lima J E, Zysler R, Duque J, Biasi E, Knobel M (2008) Effective anisotropy field variation of magnetite nanoparticles with size reduction. Eur Phys J B 64: 211-218. doi:10.1140/epjb/e2008-00294-6

Wu JH, Ko SP, Liu HL, Kim S, Ju JS, Kim YK (2007) Sub 5 nm magnetite nanoparticles: synthesis, microstructure, and magnetic properties. Mater Lett 61(1415):3124-3129. doi: 10.1016/j.matlet.2006.11.032

Yang X, Liu Q, Chen X, Zhu Z (2008) Investigation on the formation mechanisms of hydrogels made by combination of $\gamma$-ray irradiation and freeze-thawing. J Appl Polym Sci 108(2):1365-1372. doi:10.1002/app.27832

Zboril R, Mashlan M, Petridis D (2002) Iron(iii) oxides from thermal processes-synthesis, structural and magnetic properties, Mössbauer spectroscopy characterization, and applications. Chem Mater 14(3):969-982. doi:10.1021/cm01 11074

Zhao Z, Huang D, Yin Z, Chi X, Wang X, Gao J (2012) Magnetite nanoparticles as smart carriers to manipulate the cytotoxicity of anticancer drugs: magnetic control and $\mathrm{pH}-$ responsive release. J Mater Chem 22:15717-15725. doi: 10.1039/C2JM31692G

Zrínyi M, Barsi L, Szabó D, Kilian HG (1997) Direct observation of abrupt shape transition in ferrogels induced by nonuniform magnetic field. J Chem Phys 106(13): 5685-5692. doi:10.1063/1.473589 